\title{
SELLADORES BIOCERÁMICOS Y TÉCNICAS DE OBTURACIÓN EN ENDODONCIA
}

Bioceramic sealants and obturation techniques in Endodontics

Fecha de Recepción: 12 de abril 2021
Cimentos obturadores biocerámicos e técnicas obturação endodónticas

Aceptado para su publicación: 12 de mayo de 2021
1. Carrera de Especialización en Endodoncia. Facultad de Ciencias de la Salud. Universidad Católica de Córdoba. República Argentina.

2. Cátedra de Endodoncia II. Universidad del Salvador/ Asociación Odontológica (Buenos Aires). República Argentina.

3. Cátedra de Endodoncia. Facultad de Odontología. Universidad Nacional de Córdoba. República Argentina. a. Especialista en endodoncia (Sociedad Argentina de Endodoncia/MSP)

b. Doctor en Odontología (Universidad Nacional de Córdoba)

Correspondencia:

Alberdi, Jorge Carlos Buenos Aires 669. CP: 2520 Las Rosas. Santa Fe. Argentina $+5403471451434$

Correo electrónico: endojorgealberdi@gmail.com

Conflicto de intereses: los autores declaran no tener conflictos de interés.

Fuente de financiamiento: autofinanciado.

\section{Resumen}

El objetivo principal de la obturación endodóntica es rellenar de manera hermética y tridimensional el sistema de conductos radiculares con materiales inertes, antisépticos y/o bioactivos que estimulen el proceso de reparación o no lo interfieran. La utilización de agentes selladores para la obturación endodóntica es esencial para rellenar las irregularidades del conducto y las pequeñas discrepancias entre la pared dentinaria y el material sólido de obturación. En los últimos años, han surgido en el mercado una gran variedad de selladores endodónticos biocerámicos. Se han difundido en su uso clínico de manera muy vertiginosa, por sus ventajas y propiedades que favorecen sobre todo a los procesos de reparación ósea, dado que son materiales bioactivos. Se buscó literatura relacionada al tema planteado en la plataforma Pubmed y Medline para desarrollar esta revisión narrativa, con el fin de estudiar la relación entre los nuevos selladores biocerámicos y las diferentes técnicas de obturación termoplásticas aplicadas en Endodoncia. Es importante destacar la poca evidencia científica respecto del comportamiento clínico de los selladores biocerámicos durante la obturación endodóntica, más aún cuando se aplica calor como parte de la técnica.

Palabras clave: endodoncia; obturación del conducto radicular; selladores del conducto radicular (fuente: DeCS BIREME).

\section{Abstract}

The main objective of endodontic obturation is to fill the root canal system tightly and three-dimensionally with inert, antiseptic and/or bioactive materials that stimulate the repair process or do not interfere with it. The use of sealing agents for endodontic obturation is essential to fill canal irregularities and small discrepancies between the dentin wall and the solid filling mate- 
rial. In recent years, a wide variety of bioceramic endodontic sealers have emerged on the market. They have spread in their clinical use in a very vertiginous way, due to their advantages and properties that favor-above all- bone repair processes, since they are bioactive materials. Literature related to the issue was searched in Pubmed and Medline to develop this narrative review, in order to study the relationship between the new bioceramic sealants and the different thermoplastic filling techniques applied in Endodontics. It is important to highlight the little scientific evidence regarding the clinical behavior of bioceramic sealants during endodontic obturation, even more so when heat is applied as part of the technique.

Key words: endodontics; root canal obturation; root canal sealants (source: MeSH NLM).

\section{Resumo}

O objetivo principal da obturação endodôntica é preencher de forma hermética e tridimensional o sistema de canais radiculares com materiais inertes, anti-sépticos e / ou bioativos que estimulem ou não interfiram no processo de reparo. O uso de cimentos obturadores para obturação endodôntica é essencial para preencher irregularidades do canal e pequenas discrepâncias entre a parede dentinária e o material obturador sólido. Nos últimos anos, uma grande variedade de cimentos obturadores biocerâmicos surgiu no mercado. Têm se difundido em seu uso clínico de forma bastante vertiginosa, devido às suas vantagens e propriedades que favorecem principalmente os processos de reparo ósseo, por se tratar de materiais bioativos. A literatura relacionada ao tema levantado nas plataformas Pubmed e Medline foi pesquisada para desenvolver esta revisão narrativa, a fim de estudar a relação entre os novos cimentos obturadores biocerâmicos e as diferentes técnicas de obturação termoplásticas aplicadas em Endodontia. É importante destacar as poucas evidências científicas a respeito do comportamento clínico dos cimentos obturadores biocerâmicos durante obturação endodôntica, principalmente quando o calor é aplicado como parte da técnica.

Palavras-chave: endodontia; obturação do canal radicular; selantes do canal radicular (fonte: DeCS BIREME).

\section{Introducción}

El éxito del tratamiento endodóntico depende, entre otros factores, de un adecuado diagnóstico, seguido de una preparación biomecánica del sistema de conductos radiculares y la obturación tridimensional del mismo. El objetivo principal de la obturación endodóntica es rellenar de manera hermética (sellar todo tipo de comunicación conducto-peridodonto y conducto-medio bucal) y tridimensional el sistema de conductos radiculares con materiales inertes, antisépticos y/o bioactivos que estimulen el proceso de reparación o no lo interfieran ${ }^{1}$.

Los materiales habitualmente utilizados para la obturación del sistema de conductos radiculares son la gutapercha en combinación con selladores endodónticos, con el fin de obtener el relleno tridimensional del sistema de conductos. La técnica de compactación lateral es la más utilizada por la mayoría de los profesionales, por ser una técnica simple, económica y con óptimos resultados. Sin embargo, las técnicas que utilizan la gutapercha termoplastificada (por la aplicación de calor) permiten obtener una obturación más completa del espacio del conducto radicular ${ }^{2}$.

La utilización de agentes selladores para la obturación endodóntica es esencial para rellenar las irregularidades del conducto y las pequeñas discrepancias entre la pared dentinaria y el material sólido de obturación ${ }^{3}$. Un cemento sellador ideal, según Grossman debe proporcionar adhesión entre el material de obturación y la pared dentinaria, producir un sellado hermético del conducto, no contraer al fraguar, ser radiopaco, no pigmentar la estructura dentaria, ser insoluble en fluidos bucales, biocompatible y bien tolerado por los tejidos perirradiculares ${ }^{4}$.

Dentro del grupo de los selladores endodónticos, los que son a base de óxido de zinc eugenol han sido los más utilizados a nivel mundial por su adecuada plasticidad, consistencia, eficacia selladora y pequeñas alteraciones volumétricas que presentan posterior al fraguado 5 . Sin embargo, se ha demostrado la toxicidad de estos selladores en contacto con tejidos vivos, generando una respuesta inflamatoria de leve a severa en los tejidos periapicales, por el contenido del eugenol ${ }^{6}$. Además, el eugenol interfiere en la polimerización de los materiales resinosos adhesivos 
de restauración coronaria, que se utilizan posterior al tratamiento endodóntico?.

Existe también otro grupo de selladores a base de resina epóxica, que no contienen eugenol, y han sido introducidos en la práctica endodóntica por sus ventajas de adhesión a la estructura dentaria, adecuado tiempo de trabajo, fácil manipulación y buen sellado ${ }^{8}$. AH-Plus ${ }^{\circledR}$ (Dentsply Maillefer) es un sellador compuesto por resina epóxica y aminas, introducido en el mercado en 1997. Se ha demostrado que $\mathrm{AH}$ Plus ${ }^{\circledR}$ presenta adecuado sellado, estabilidad dimensional, alta radiopacidad y polimerización sin formación de formaldehído y propiedades autoadhesivas ${ }^{9}$. Un estudio histopatológico sobre la respuesta del tejido subcutáneo de la rata a tres selladores endodónticos, reveló que el sellador de Grossman tuvo la respuesta inflamatoria más severa seguida por AH Plus y Epiphany ${ }^{10}$.

En los últimos años, han surgido en el mercado una gran variedad de selladores endodónticos biocerámicos. Se han difundido en su uso clínico de manera muy vertiginosa, por sus ventajas y propiedades que favorecen sobre todo a los procesos de reparación ósea, dado que son materiales Bioactivos. La mayor parte de la bibliografía y de los estudios se refieren a los materiales biocerámicos usados como cemento reparador, o sea tratamiento de perforaciones, tapones apicales, entre otros, desde la aparición de los trióxidos minerales agregados (MTA) en el año 1999.

Si bien, los selladores biocerámicos tienen de base la misma composición (silicato tricálcico) que los cementos biocerámicos, para poder reunir las características y consistencia de un sellador endodóntico se le adicionan otros componentes que podrían hacer que éste se comporte de manera diferente al de los cementos biocerámicos reparadores.

El objetivo de esta revisión narrativa es investigar en la literatura, la relación entre los nuevos selladores biocerámicos y las diferentes técnicas de obturación termoplásticas aplicadas en Endodoncia.

\section{Metodología}

Estrategia y criterios de búsqueda. Se buscó literatura de los últimos 10 años en la plataforma Pub- med y Medline sobre los diferentes tópicos: obturación endodóntica, selladores endodónticos, MTA y selladores biocerámicos, técnicas de obturación y selladores biocerámicos, aplicación de calor a los selladores biocerámicos.

Criterios de selección: artículos sobre selladores biocerámicos publicados desde el año 2010 a la actualidad y artículos sobre obturación endodóntica.

\section{Selladores biocerámicos: contexto histórico}

En 1993 se desarrolló el primer cemento biocerámico a base de trióxido mineral agregado (MTA, ProRoot Dentsply) para el sellado de perforaciones accidentales del conducto radicular ${ }^{11}$. MTA es un cemento bioactivo con capacidad para inducir la formación de nuevo tejido mineralizado, lo que hace que este material sea biológicamente aceptable para retro obturaciones en cirugías apicales, y para el tratamiento de apexificación y endodoncia regenerativa en dientes con ápices inmaduros ${ }^{12}$. Su mecanismo de acción se basa en la liberación de iones calcio que activan la adhesión y proliferación celular, y por su pH elevado se crea un medio antibacteriano (Porter et al. 2010). Posteriormente, aparecieron en el mercado otras formas comerciales, como MTA Angelus ${ }^{\circledR}$, con similares propiedades químicas al MTA conteniendo bismuto para aumentar su radiopacidad $^{13}$

En el año 2009 se presenta Biodentine ${ }^{\circledR}$ (Septodont, SaintMaur-des-Fosses, Francia) un cemento bioactivo, sustituto de la dentina, compuesto principalmente por silicato tricálcico ${ }^{14}$. Por sus propiedades biológicas y físicas mejoradas, Biodentine presenta una resistencia a la compresión significativamente mayor que MTA White ${ }^{15}$. Además, su microdureza, resistencia a la flexión, capacidad de sellado, fuerza de adhesión y liberación de iones de calcio son notablemente superiores en comparación con otros cementos a base de silicato tricálcico ${ }^{16}$. Una de las ventajas de Biodentine es que el tiempo de fraguado es de 15 minutos, resultando más corto respecto al de MTA que fragua en 4 horas $^{17}$ y su estabilidad del color en el tiempo ${ }^{18}$.

Posteriormente, se desarrollaron los selladores a base de biocerámicos, indicados para la obturación permanente del conducto radicular en combinación con conos de gutapercha, mediante la técnica de 
cono único o compactación lateral en frío ${ }^{19}$. BioRoot RCS (Septodont, SaintMaur-des-Fosses, Francia) se presenta en forma de polvo y líquido. El polvo contiene óxido de circonio como radiopacificador y un polímero biocompatible hidrofílico para mejorar la adherencia. El líquido contiene principalmente agua y cloruro de calcio lo cual acelera el fraguado. BioRoot RCS mostró un sellado hermético con la dentina y la gutapercha, y una radiopacidad adecuada ${ }^{20}$.

\section{Selladores biocerámicos: biocompatibilidad y bioactividad}

Los selladores biocerámicos son bioactivos porque estimulan el proceso fisiológico del hueso y la mineralización de la estructura dentinaria ${ }^{21}$. Por sus propiedades bioactivas tales como la biocompatibilidad, la formación de hidroxiapatita, mineralización de la estructura dentinaria, $\mathrm{pH}$ alcalino y propiedades de sellado, se crea un entorno favorable para la cicatrización periapical ${ }^{22}$. El calcio, el silicio y el carbonato difunden en los túbulos dentinarios, lo que resulta en la formación de una "zona de infiltración marginal", otorgando alta resistencia a las microfiltraciones y anclaje micromecánico mediante la precipitación en la entrada de los túbulos dentinarios. Al igual que otros cementos biocerámicos, no se reabsorbe, no se contrae, sino que posee una pequeña expansión durante su fraguado ${ }^{23}$.

Recientemente, apareció en el mercado Bio-C Sealer (Angelus, Brasil), un sellador biocerámico que contiene silicato de calcio, aluminato de calcio y óxido de calcio, lo cual hace que este material sea biocompatible y bioactivo, por la liberación de iones calcio. Además, contiene óxido de zirconio, óxido de hierro, dióxido de silicio y propilenglicol como agente de dispersión, sin sufrir contracción posterior al fraguado. Las propiedades mecánicas y físicas confieren facilidad en el manejo y sellado hermético de los conductos, además de sus propiedades biológicas y $\mathrm{pH}$ elevado ( $\mathrm{pH}: 12,5)$. Puede ser utilizado, con la técnica de compactación lateral, cono único y obturación termoplástica (según las indicaciones del fabricante), para la obturación de conductos radiculares de dientes permanentes y tratamiento de la reabsorción dentinaria interna. También existen selladores de otras marcas con características similares como Ceraseal (Meta), Totalfill (FKG) con similares características a estos últimos y con las mismas indicaciones.

\section{Técnicas de obturación termoplásticas}

La obturación del sistema de conductos involucra, como ya fue descripto anteriormente, el uso de gutapercha como material principal y selladores endodónticos con el fin de ocupar los espacios libres que no llega a rellenar el material principa ${ }^{24}$. Las técnicas termoplásticas de obturación han sido desarrolladas para que la gutapercha ocupe más espacio dentro sistema de conductos y por consiguiente lograr una obturación tridimensional ${ }^{25}$.

Las técnicas más utilizadas para plastificar la gutapercha son 2: la Técnica de compactación vertical caliente y la Técnica de obturación a base un carrier, como son Thermafil o Guttacore y similares.

Al plastificar la gutapercha con las técnicas de compactación vertical, la gutapercha pasa de una fase beta (fría) a una fase alfa (plastificada) para luego volver a ser beta, utilizando los dispositivos ya mencionados con sus diferentes atacadores calientes calibrados según el taper del conducto a obturar. El calentamiento y posterior enfriamiento que se produce durante la aplicación de los atacadores podría alterar la consistencia o propiedades de los selladores endodónticos.

Existen estudios que describen la solubilidad y la desintegración de los distintos selladores endodónticos, por lo cual sería recomendable que la obturación del conducto radi-cular contenga, en su mayor proporción gutapercha, acompañada por sólo una película superficial de sellador. Esto se debe a la estabilidad dimensional y durabilidad en el tiempo de la gutapercha ${ }^{26,27}$.

Se han desarrollado diferentes técnicas con el fin de plastificar la gutapercha y poder compactarla en el interior de los conductos radiculares, haciendo de este modo que éste se rellene principalmente con gutapercha, y sea necesario una menor cantidad de sellador endodóntico. La importancia de reducir la cantidad de sellador estaría justificada, ya que se ha demostrado que la filtración bacteriana ocurriría en la interface sellador y gutapercha o entre el sellador y la pared dentinaria ${ }^{28}$.

Estudios recientes indican que el modelo de estudio para evaluar los cambios de temperatura 
cuando se emplean estas técnicas, se vuelve un factor principal para poder trasladar los resultados a la clínica. En un sistema cerrado con tejidos circundantes simulados a $37^{\circ} \mathrm{C}$, la técnica de onda contínua produjo mayores aumentos de temperatura que la técnica de relleno con gutapercha fluida con un máximo de $19.1^{\circ} \mathrm{C}$. El aumento de temperatura durante la obturación con Thermafil fue insignificante. El aumento de temperatura en el sellador endodóntico fue marcadamente más bajo de lo esperado en los estudios de medición de temperatura de la superficie radicular ${ }^{29}$.

Cuando se compararon ambas técnicas termoplásticas, a base de un carrier u obturador y compactación vertical caliente, se demostró que ambas fueron igualmente eficientes en relación con la adaptación apical. No hubo diferencias significativas con respecto al porcentaje de huecos o espacios de aire apicales independientemente del nivel del conducto evaluado ${ }^{30}$. Dentro de los parámetros de una evaluación ex vivo, el sistema Thermafil produjo áreas rellenas con gutapercha significativamente mayores que la condensación lateral o los grupos del System B. Esto significó una reducción en la cantidad de sellador y se propone esta técnica a base de un carrier especialmente en conductos radiculares con formas irregulares ${ }^{31}$.

La compatibilidad de un material de obturación con la técnica de obturación utilizada no es menos importante que cualquier paso del tratamiento endodóntico, en el cual la integridad, durabilidad y biocompatibilidad de la obturación del conducto radicular son esenciales para alcanzar el éxito. Pocos estudios evaluaron este aspecto de la obturación, entre ellos, Camilleri ${ }^{19}$, en 2015 estudió la alteración de tres selladores a base de resina y un sellador a base de silicato tricálcico (en experimentación) y observó que el uso de sellador experimental a base de silicato tricálcico para obturaciones dio mejores resultados cuando se utilizó gutapercha fría condensada lateralmente. $Y$ respecto a los selladores a base de resina propuso que deben usarse con técnicas de obturación de gutapercha termoplastizada. Contrariamente, Atmeh ${ }^{32}$, en 2017 observó que el calentamiento no causó cambios detectables en la estructura química del sellador a base de silicato de calcio, aunque pueden ocurrir cambios microestruc- turales debido a la pérdida de agua. La aplicación de calor en el sellador a base de resina epoxi dentro del rango de temperatura recomendado durante 30 segundos indujo cambios mínimos en la estructura química. Sin embargo, el calentamiento a temperaturas más altas y / o una mayor duración se asoció con una polimerización más temprana con cambios en la estructura química de los monómeros epoxi, los endurecedores de amina y la carga de tungstato de calcio. Estos cambios fueron dependientes de la temperatura y el tiempo, y estos últimos tuvieron un mayor impacto. Más recientemente, Heran et al. ${ }^{33}$, estudiaron BioRoot sealer, Ah PLus y Guttaflow y determinaron que la aplicación de calor afectó las propiedades del sellador y el volumen vacío. Además, sugieren que AH Plus no debe someterse a altas temperaturas dado que sus propiedades se deterioran y el volumen vacío aumentó. Adicionalmente, observaron que la obturación con cono único puede no ser adecuada para la obturación de anatomías complejas. Por otra parte, Qu et al. ${ }^{34}$, revelaron que el sellador a base de ZOE y el sellador a base de resina AH Plus mostraron cambios aceptables en las propiedades físicas (tiempo de fraguado y flujo) a una temperatura alta. Sin embargo, se encontraron reducciones significativas en el tiempo de fraguado y el flujo en el sellador a base de silicato tricálcico biocerámico iRoot SP y el sellador a base de silicato RoekoSeal, lo que podría afectar negativamente el rendimiento de la práctica clínica y la calidad de la obturación con la técnica de compactación vertical caliente.

Con respecto a las técnicas basadas en obturadores o carriers, un reciente trabajo de Ahmed et al. ${ }^{35}$, destacó la importancia de identificar los niveles reales de temperatura de los carriers de obturación por parte de los profesionales, y la idoneidad de conocer los selladores que van a ser utilizados a la temperatura alcanzada. Todos los obturadores analizados alcanzaron niveles de temperatura inferiores a $60^{\circ} \mathrm{C}$ cuando se usaron en modo de desconexión, que es un nivel seguro para la gutapercha y todos los selladores. Sin embargo, el uso de algunos dispositivos que utilizan una onda contínua de calor puede producir una temperatura más alta que puede exceder los niveles de seguridad sugeridos. Esto puede arriesgar la integridad de los materiales de obturación del conducto radicular. Es posible que 
no se recomiende que los selladores a base de óxido de zinc se calienten debido al efecto perjudicial del calor sobre sus estructuras moleculares. La resina epoxi y los selladores a base de silicato de calcio sometidos a prueba soportaron calor por debajo de $100^{\circ} \mathrm{C}$, sin embargo, no se recomienda una temperatura más alta.

\section{Conclusiones}

Estos materiales constituyen una alternativa de gran interés en la obturación endodóntica. Sin embargo, es importante destacar la poca evidencia científica respecto de su comportamiento clínico sobre cómo durante la obturación endodóntica podría afectarse alguna función o propiedad física de los mismos, más aún cuando se aplica calor como parte de la técnica.

\section{Referencias Bibliográficas}

1. Soares I, Goldberg F. Endodoncia: Técnicas y fundamentos. 2da ed. Buenos Aires: Editorial Médica Panamericana; 2012.

2. Goldberg F, Araujo J. Estudio comparativo de la penetración de la gutapercha en las irregularidades del conducto radicular. Sistema Gutta-Core y técnica de cono único. Rev Asoc Odontol Argent. 2016;104(1):4-8.

3. Cohen S; Hargreaves K. Vías de la Pulpa. $11^{\circ}$ Edición. España: Ed. Elsevier;2016.

4. Zhou H, Shen Y, Zheng W, Li L, Zheng Y, Haapasalo M. Physical properties of 5 root canal sealers. J Endod. 2013; 39(10):1281-1286. doi: 10.1016/j.joen.2013.06.012

5. Tyagi S, Tyagi P, Mishra P. Evolution of root canal sealers: An insight story. Eur J Gen Dent. 2013; 2(3):199-218. doi: 10.4103/2278-9626.115976

6. Araki K; Suda H; Spangberg L. Indirect longitudinal cytotoxicity of root canal sealers L929 cells and human periodontal ligament fibroblasts. J Endod. 1998; 20 (1): 67-70.

7. Marín-Bauza GA, Silva-Sousa YTC, Cunha SA da, Rached-Junior FJA, Bonetti-Filho I, Sousa-Neto MD et al . Physicochemical properties of endodontic sealers of different bases. J Appl Oral Sci. 2012; 20(4):455-461. doi: 10.1590/S1678-77572012000400011

8. Resende LM, Rached-Junior FJ, Versiani MA, Souza-Gabriel AE, Miranda CE, Silva-Sousa YT et al. A comparative study of physicochemical properties of $\mathrm{AH}$ plus, epiphany, and epiphany SE root canal sealers. Int Endod J. 2009;42(9):785-793. doi: 10.1111/j.13652591.2009.01584.x

9. Hubbe L, Oliveira D, Vianna K, Coelho S. AH Plus extrusion into periapical tissue: literature 74 review of main related properties and report of clinical cases. RSBO.2016;13(4):280-288. Disponible en: https://www. redalyc.org/pdf/1530/153050677011.pdf

10. Farhad AR, Hasheminia S, Razavi S, Feizi M. Histopathologic evaluation of subcutaneous tissue response to three endodontic sealers in rats. J Oral Sci 2011;53(1):1521. doi: $10.2334 /$ josnusd.53.15

11. Lee S, Monsef M, Torabinejad M. Sealing ability of mineral trioxide aggregate for repair of lateral root perforations. J Endod. 1993;19(11):541-4. doi: 10.1016/S00992399(06)81282-3

12. Bakland L, Andreasen J. Will mineral trioxide aggregate replace calcium hydroxide in treating pulpal and periodontal healing complications subsequent to dental trauma? A review. Dent Traumatol. 2012;28(1):25-32. doi: 10.1111/j.1600-9657.2011.01049.x

13. Oliveira M, Xavier C, Demarco F, Pinheiro A, Costa A, 
Revista de la Facultad de Odontología

Selladores biocerámicosy técnicas de obturación en endodoncia

\section{REFO}

VOL. XIV | No 1| AÑO 2021

ISSN 1668-7280

ISSN-E 2683-7986
Pozza D. Comparative chemical study of MTA and Portland cements. Braz Dent J. 2007; 18(1):3-7. doi: 10.1590/ s0103-64402007000100002

14. Camilleri J, Sorrentino F, Damidot D. Investigation of the hydration and bioactivity of radiopacified tricalcium silicate cement, Biodentine and MTA Angelus. Dental Materials 2013;29(5):580-593. doi: 10.1016/j.dental.2013.03.007

15. Elnaghy, A. M. Influence of Acidic Environment on Properties of Biodentine and White Mineral Trioxide Aggregate: A Comparative Study. J Endod. 2014; 40(7): 953957. doi: 10.1016/j.joen.2013.11.007

16. Rajasekharan S, Martens L, Cauwels R, Anthonappa R. Biodentine material characteristics and clinical applications: a 3 year literature review and update. Eur Arch Paediatr Dent.2018;19(1):1-22. doi: 10.1007/s40368018-0328-x

17. Darvell BW, WU RCT. "MTA"-An Hydraulic Silicate Cement: Review update and setting reaction. Dental Materials 2011;27(5):407-422. doi: 10.1016/j.dental.2011.02.001

18. Kohli MR, Yamaguchi M, Setzer FC, Karabucak B. Spectrophotometric Analysis of Coronal Tooth Discoloration Induced by Various Bioceramic Cements and Other Endodontic Materials. J Endod 2015;41(11):1862-1866. doi: 10.1016/j.joen.2015.07.003

19. Camilleri J. Sealers and warm gutta-percha obturation techniques. J Endod. 2015; 41(1):72-8. doi: 10.1016/j. joen.2014.06.007

20. Xuereb M, Vella P, Damidot D, Sammut CV, Camilleri J. In Situ Assessment of the Setting of Tricalcium Silicatebased Sealers Using a Dentin Pressure Model. J Endod. 2015;41(1):111-124. doi: 10.1016/j.joen.2014.09.015

21. Camps J, Jeanneau C, El Ayachi I, Laurent P, About I. Bioactivity of a Calcium Silicate-based Endodontic Cement (BioRoot RCS): Interactions with Human Periodontal Ligament Cells In Vitro. J Endod. 2015;41(9):14691473. doi: 10.1016/j.joen.2015.04.011

22. Dimitrova-Nakov S, Uzunoglu E, Ardila-Osorio $\mathrm{H}$, Baudry A, Richard G, Kellermann O, Goldberg M. In vitro bioactivity of Bioroot ${ }^{\mathrm{TM}}$ RCS, via A4 mouse pulpal stem cells. Dent Mater. 2015;31(11):1290-1297. doi: 10.1016/j.dental.2015.08.163

23. Viapiana R, Guerreiro-Tanomaru J, Tanomaru-Filho $\mathrm{M}$, Camilleri J. Interface of dentine to root canal sealers. Journal of Dentistry. 2014;42(3):336-350. doi: 10.1016/j. jdent.2013.11.013

24. Schafer E, Olthoff G. Effect of Three Different Sealers on the Sealing Ability of Both Thermafil Obtura- tors and Cold Laterally Compacted Gutta-Percha. J Endod. 2002;28(9):638-642. doi: 10.1097/00004770200209000-00003

25. Schilder $\mathrm{H}$. Filling root canals in three dimensions. Dent Clin North Am. 1967;723-44.

26. Kaplan AE, Goldberg F, Artaza LP, De Silvio A, Macchi RL. Disintegration of endodontic cements in water. J Endod. 1997;23(7):439-ح41. doi: 10.1016/S00992399(97)80298-1

27. Tay FR, Pashley DH, Loushine RJ, Kuttler S, Garcíaっ Godoy F, King NM, Ferrari M. Susceptibility of a polycaprolactone $\neg$ base canal filling material to degradation. Evidence of biodegrada $\neg$ tion from a simulated field test. Am J Dent. 2007;20(6):365-ᄀ9.

28. Kontakiotis EG, Wu MK, Wessilink PR. Effect of sealer thickness on long-term sealing ability: a two year follow-up study. Int Endod J. 1997;30(5):307-12. doi: 10.1046/j.1365-2591.1997.00087.x

29. Donnermeyer D, Schäfer E, Bürklein S. Real-time intracanal temperature measurement during different obturation techniques. J Endod. 2018;44(12):1832-6. doi: 10.1016/j.joen.2018.08.013

30. Zogheib C, Hanna M, Pasqualini D, Naaman A. Quantitative volumetric analysis of cross-linked gutta-percha obturators. Ann Stomatol. (Roma) 2017;7(3):46-51. doi:10.11138/ads/2016.7.3.046

31. De-Deus G, Maniglia-Ferreira C, Gurgel-Filho E, Paciornik S, Machado A, Coutinho-Filho T. Comparison of the percentage of gutta-percha-filled area obtained by Thermafil and System B. Aust Endod J. 2007; 33(2): 55-61. doi:10.1111/j.1747-4477.2007.00047.x

32. Atmeh AR, Alshwaimi E. The effect of heating time and temperature on epoxy resin and calcium silicate-based endodontic sealers, J Endod. 2017; 43(12): 2112-2118. doi: 10.1016/j.joen.2017.08.008

33. Heran J, Khalid S, Albaaj F, Tomson PL, Camilleri J. The single cone obturation technique with a modified warm filler. J Dent. 2019; 89:10318. doi: 10.1016/j. jdent.2019.103181

34. Qu W, Bai W, Liang YH, Gao XJ. Influence of Warm Vertical Compaction Technique on Physical Properties of Root Canal Sealers. J Endod. 2016;42(12):1829-1833. doi: 10.1016/j.joen.2016.08.014

35. Atmeh AR, Hadis M, Camilleri J. Real-time chemical analysis of root filling materials with heating: guidelines for safe temperature levels. Int Endod J. 2020;53(5):698708. doi: 10.1111/iej.13269 